\title{
EPIDEMIOLOGY \\ Lipid level trends improving in USA
}

Serum levels of total cholesterol, LDL cholesterol, non-HDL cholesterol and triglycerides have decreased in the US adult population in the past 22 years, which was accompanied by increases in serum levels of HDL cholesterol, reveals an analysis of data from three National Health and Nutrition Examination Surveys (NHANES). Such improvements are hopeful news in the fight against atherosclerosis and coronary heart disease.

Margaret Carroll and colleagues at the Centers for Disease Control and Prevention and $\mathrm{NIH}$, Maryland, USA, analysed data from adults aged $\geq 20$ years who took part in the 1988-1994 ( $n=16,573), 1999-2002$ $(n=9,471)$ or the $2007-2010(n=11,766)$ NHANES. These groups constitute representative samples of the noninstitutionalized US population. A small proportion of participants was excluded from the analysis owing to missing data.

Encouragingly, the researchers observed statistically significant declines in total cholesterol, LDL cholesterol and non-HDL cholesterol levels over the studied 22-year period. "The percentage of adults receiving lipidlowering medications continued to increase during this period," Carroll points out, "which most likely contributed to the decline in total and LDL cholesterol levels." John Kastelein (Academic Medical Center, University of Amsterdam, the Netherlands), who was not involved in the study, comments that the majority of these lipid-lowering drugs are probably statins. "The fact that lipid-lowering drugs potentially have such an effect at a population level is amazing," he adds, stressing the positive effect that such an improvement can have on the incidence of coronary heart disease at a population level.

"The increase in the use of cholesterol-lowering medications is not the only explanation for the declining trends in total and LDL cholesterol levels during 1988-2010," says Carroll, who observed decreasing total cholesterol and LDL-cholesterol levels among adults not receiving this type of therapy. "A decline in dietary intake of trans fatty acids, which affects trans-fatty-acid levels in blood, could also have contributed to the decline in total and LDL cholesterol levels," she explains.

HDL cholesterol levels increased in the studied population as a whole and among non-Hispanic white adults of both sexes between 1988 and 2010, although not in Mexican American or non-Hispanic black adults of either sex. Triglyceride levels, on the other hand, increased between 1988 and 2002, but then decreased until 2010 in all adults and among individuals of either sex. "The decrease in the percentage of cigarette smokers could be one explanation for these favourable trends in HDL cholesterol and triglyceride levels," says Carroll.

The reported data, in Kastelein's opinion, will probably be used to support an increased prescription of statins in clinical practice, especially as these medications are generic; they might also be used to support the implementation of the 'polypill' approach. "There was not much proof that lipid-lowering medications could have such a strong effect at the population level, but these data, although not from a randomized trial, make a very strong case to act," says Kastelein. "Unpublished population data from the UK indicate that cholesterol levels have been decreasing from the mid1990s and over the same period a decrease in the incidence of coronary heart disease was also observed. These observations will strengthen the NHS decision to reward general practitioners for keeping their patients below a certain cholesterol goal."

Carroll and colleagues plan to continue monitoring trends in total and LDL cholesterol levels, as well as trends in HDL cholesterol and triglyceride levels in the US population. "We also intend to look at apoB, virtually the only protein component of LDL cholesterol, which could provide useful information with respect to assessing risk of coronary heart disease."

Joana Osório

Original article Carroll, M. D. et al. Trends in lipids and lipoproteins in US adults, 1988-2010. JAMA

doi:10.1001/jama.2012.13260 